\title{
Live imaging the phagocytic activity of inner ear supporting cells in response to hair cell death
}

\author{
EL Monzack ${ }^{1}$, LA May ${ }^{1}$, S Roy ${ }^{1}$, JE Gale ${ }^{2}$ and LL Cunningham ${ }^{\star, 1}$
}

Hearing loss and balance disorders affect millions of people worldwide. Sensory transduction in the inner ear requires both mechanosensory hair cells (HCs) and surrounding glia-like supporting cells (SCs). HCs are susceptible to death from aging, noise overexposure, and treatment with therapeutic drugs that have ototoxic side effects; these ototoxic drugs include the aminoglycoside antibiotics and the antineoplastic drug cisplatin. Although both classes of drugs are known to kill HCs, their effects on SCs are less well understood. Recent data indicate that SCs sense and respond to HC stress, and that their responses can influence $\mathrm{HC}$ death, survival, and phagocytosis. These responses to $\mathrm{HC}$ stress and death are critical to the health of the inner ear. Here we have used live confocal imaging of the adult mouse utricle, to examine the SC responses to HC death caused by aminoglycosides or cisplatin. Our data indicate that when HCs are killed by aminoglycosides, SCs efficiently remove HC corpses from the sensory epithelium in a process that includes constricting the apical portion of the HC after loss of membrane integrity. SCs then form a phagosome, which can completely engulf the remaining HC body, a phenomenon not previously reported in mammals. In contrast, cisplatin treatment results in accumulation of dead HCs in the sensory epithelium, accompanied by an increase in SC death. The surviving SCs constrict fewer HCs and display impaired phagocytosis. These data are supported by in vivo experiments, in which cochlear SCs show reduced capacity for scar formation in cisplatin-treated mice compared with those treated with aminoglycosides. Together, these data point to a broader defect in the ability of the cisplatin-treated SCs, to preserve tissue health in the mature mammalian inner ear.

Cell Death and Differentiation (2015) 22, 1995-2005; doi:10.1038/cdd.2015.48; published online 1 May 2015

Hearing loss affects more than 360 million people worldwide and is often irreversible. ${ }^{1}$ Mechanosensory hair cells (HCs), the receptor cells of hearing and balance, are not regenerated in the adult mammal and their death results in permanent hearing loss. ${ }^{2,3} \mathrm{HCs}$ are surrounded by glia-like supporting cells (SCs) that are necessary for $\mathrm{HC}$ survival and function (reviewed in Monzack et al.). ${ }^{4}$ SCs perform many functions, including providing critical trophic factors, preventing excitotoxicity, and mediating regeneration in those systems (non-mammalian vertebrates) capable of replacing lost HCs. ${ }^{5-11}$ When HCs die, SCs also preserve the integrity and function of the remaining tissue by forming scars and clearing dead HCs. ${ }^{2,12-17}$ Maintaining a fluid barrier at the surface of the sensory epithelium after damage is necessary to preserve the electro-chemical gradient that drives $\mathrm{HC}$ depolarization and therefore sensory transduction after the onset of hearing (reviewed in Wangemann). ${ }^{18}$

Several major stressors cause $\mathrm{HC}$ death, ${ }^{19-22}$ including aging, noise trauma, and exposure to therapeutic drugs with ototoxic side effects. When a $\mathrm{HC}$ is killed by noise or aminoglycoside antibiotics, surrounding SCs form a filamentous actin (F-actin) cable that constricts the $\mathrm{HC}$ at its apex. ${ }^{2,12-17}$ This process separates the apical portion of the cell, including the stereocilia bundle, from the $\mathrm{HC}$ body and preserves a sealed reticular lamina. ${ }^{23}$ In the chick utricle, following the apical constriction of dead HCs, the SCs engulf and phagocytose the remaining $\mathrm{HC}$ corpse. ${ }^{15}$ Additional data from the chick indicate that the ototoxic drug cisplatin impairs some SC functions, including regeneration of $\mathrm{HCs}$ or clearance of $\mathrm{HC}$ debris. ${ }^{24}$ We hypothesized that SCs would have significant phagocytic activity in the mature mammalian inner ear, and that cisplatin would impair this activity. To examine these dynamic processes, we live-imaged SC phagocytic activity in the adult mouse utricle and compared the SC responses with $\mathrm{HC}$ stress and death caused by aminoglycosides versus cisplatin.

\section{Results}

Loss of tdTomato signals death. Our live-imaging experiments use whole-organ utricle cultures from adult transgenic mice that express tdTomato in all HCs. In initial experiments, dying HCs consistently lost tdTomato fluorescence and we examined whether loss of tdTomato is a reliable indicator of $\mathrm{HC}$ death. Live-imaging experiments were conducted with TOTO-3 iodide, a plasma membrane-impermeant dye and a dead cell indicator. ${ }^{15,25}$ Figure 1a shows a field of tdTomato-positive (red) HCs cultured in the aminoglycoside antibiotic neomycin as imaging begins, with the subsequent

\footnotetext{
${ }^{1}$ National Institute on Deafness and Other Communication Disorders, National Institutes of Health, Bethesda, MD, USA and ${ }^{2}$ UCL Ear Institute, University College, London WC1X 8EE, UK

*Corresponding author: L L Cunningham, National Institute on Deafness and Other Communication Disorders, National Institutes of Health, Building 35A Room 1D971, 35A Convent Drive, Bethesda, MD 20892, USA. Tel: +1 301443 2766; E-mail: lisa.cunningham @nih.gov

Abbreviations: ABR, auditory brainstem response; F-actin, filamentous actin; HC, hair cell; MIP, maximum intensity projection; ROI, region of interest; SC, supporting cell; SPB, Sorensen's phosphate buffer

Received 24.11.14; revised 20.2.15; accepted 04.3.15; Edited by M Piacentini; published online 01.5.15
} 



Figure 1 Loss of tdTomato signals HC death. (a) HCs (red) at the onset of live imaging before the addition of neomycin. Maximum intensity projection (MIP), scale bar $=10 \mu \mathrm{m}$. (b) Culture in neomycin for $16 \mathrm{~h}$ results in death of many HCs. Box indicates the HCs that are shown at higher magnification in $\mathbf{d}-\mathbf{k}$. (c) Schematic of the boxed region from $\mathbf{b}$. Two $\mathrm{HCs}$ are visible in the center of the field. SCs completely surround each $\mathrm{HC}$ in the utricle, but are not expressing any fluorophore and are therefore not visible in the image series. (d-k) TOTO-3 was added to cultures as a dead cell indicator, following $19 \mathrm{~h}$ of imaging in neomycin $\left(\mathrm{t}_{0}\right)$. Gray arrow indicates a TOTO-3-positive HC nucleus that lost membrane integrity before the addition of TOTO-3. Scale bar $=2 \mu \mathrm{m}$. (h and i) The HC outlined in yellow (HC) loses tdTomato signal between +80 and +90 min. (i and j) The TОTO-3 signal in this nucleus increases over the next 20 min of imaging, becoming strongly visible. The nucleus from the tdTomato-positive HC that does not die in this image series does not show an increase in TOTO-3 (magenta arrow). (I) The mean TOTO-3 fluorescent intensity of each indicated nucleus (above) was quantified and its change over time was plotted. The black arrow marks the last tdTomato-positive time point of the HC of interest. ( $\mathbf{m}$ ) In a separate set of experiments, TOTO-3 signal intensity was quantified for 60 min before and after $\mathrm{HC}$ death (loss of tdTomato) in neomycin. On average, cells became tdTomato-negative and TOTO-3-positive in the same 10-min imaging interval. Movie available as Supplementary Figure 1. $\mathrm{N}=26-28 \mathrm{HCs}$ across 6 image fields from 2 utricles per time point; values represent mean \pm S.E.M. 
loss of $\mathrm{HCs}$ evident after $16 \mathrm{~h}$ (Figure 1b). The inset in Figure $1 \mathrm{~b}$ highlights two HCs further analyzed following the addition of TOTO-3. Figure 1c illustrates the orientation of these two $\mathrm{HCs}$ and schematizes the orientation of the surrounding SCs, which were not visible during imaging. Figure 1d (movie in Supplementary Figure 1) shows a 3D rendering of the same two $\mathrm{HCs}$ after $19 \mathrm{~h}$ in neomycin, at which point TOTO-3 was added $\left(t_{0}\right)$. The $\mathrm{HC}$ of interest $(\mathrm{HC}$, yellow outline) lost tdTomato signal between +80 and +90 min (Figures $1 \mathrm{~h}$ and $\mathrm{i}$ ), the same period during which TOTO-3 signal intensity increased suddenly in its nucleus (black arrow on yellow line, Figure 1I). TOTO-3 signal intensity further increased over the next $10 \mathrm{~min}$ (yellow arrow, Figure 1j). Sixty minutes after the addition of TOTO-3 (Figure 1f), an adjacent cell nucleus that was tdTomato-negative at the time of TOTO-3 addition began to show TOTO-3 uptake (gray arrow, Figure 1f), which increased over time as TOTO-3 continued to diffuse into the tissue. During this same period, the neighboring $\mathrm{HC}$ showed stable tdTomato signal with no visible nuclear TOTO-3 signal (pink arrow). Figure 1I shows TOTO-3 intensity over time for each of these three cells, revealing (i) TOTO-3 diffusion into the nucleus of a tdTomatonegative $\mathrm{HC}$ (gray line), (ii) the sudden increase in TOTO-3 signal in the nucleus of the $\mathrm{HC}$ that loses tdTomato (yellow line), and (iii) the low level of nuclear TOTO-3 in the tdTomatopositive $\mathrm{HC}$ (pink line). $\mathrm{HC}$ population data show that uptake of TOTO-3 occurs in the same 10-min imaging interval as the loss of tdTomato (Figure $1 \mathrm{~m}$ ). Together, these data indicate that loss of tdTomato from the $\mathrm{HC}$ signals loss of membrane integrity and therefore $\mathrm{HC}$ death.

\section{SCs constrict and remove HCs killed by neomycin.}

To examine the $\mathrm{SC}$ response to $\mathrm{HC}$ death in the mature mammalian inner ear, we used live imaging to observe the phagocytic removal of dead HCs. Utricles were infected with Ad-LifeAct-EGFP, driving an EGFP-tagged F-actin-binding peptide in a subset of SCs. ${ }^{26,27}$ Figure 2 shows images selected from a live-imaging experiment in neomycin (movies in Supplementary Figures 2 and 3). One tdTomato-positive $\mathrm{HC}$ (red, HC1) and one LifeAct-EGFP-expressing SC (green, SC1) are labeled in Figure 2a, with the orthogonal view displayed in Figure 2a'. HC1 subsequently dies (loses tdTomato signal) at $+6 \mathrm{~h}$ (Figures $2 \mathrm{~d}$ and $\mathrm{d}$ '), followed by phagocytic activity in the neighboring SC. By $+6.5 \mathrm{~h}$, the green SC has enriched F-actin (arrowheads, Figures $2 \mathrm{e}$ and e'), constricting the apical region of the dead $\mathrm{HC}$. By $+15 \mathrm{~h}$, the SC has formed an F-actin-rich basket-like phagosome (arrowheads, Figures $2 f$ and f') surrounding the remaining $\mathrm{HC}$ corpse. The F-actin-rich phagosome has also pushed the $\mathrm{HC}$ corpse deeper into the sensory epithelium. Magnified inset images (Figure 2a") show a $\mathrm{HC}$ (HC2) and two neighboring SCs (SC2 and SC3). Following the death of $\mathrm{HC} 2$ at $+4 \mathrm{~h}$ (Figure 2b"), two adjacent SCs have constricted the tdTomato-negative $\mathrm{HC}$ corpse within $30 \mathrm{~min}$, entering the space previously occupied by the HC (Figure 2c"). The position of the tdTomato-negative $\mathrm{HC}$ remains relatively stable for several hours following loss of membrane integrity
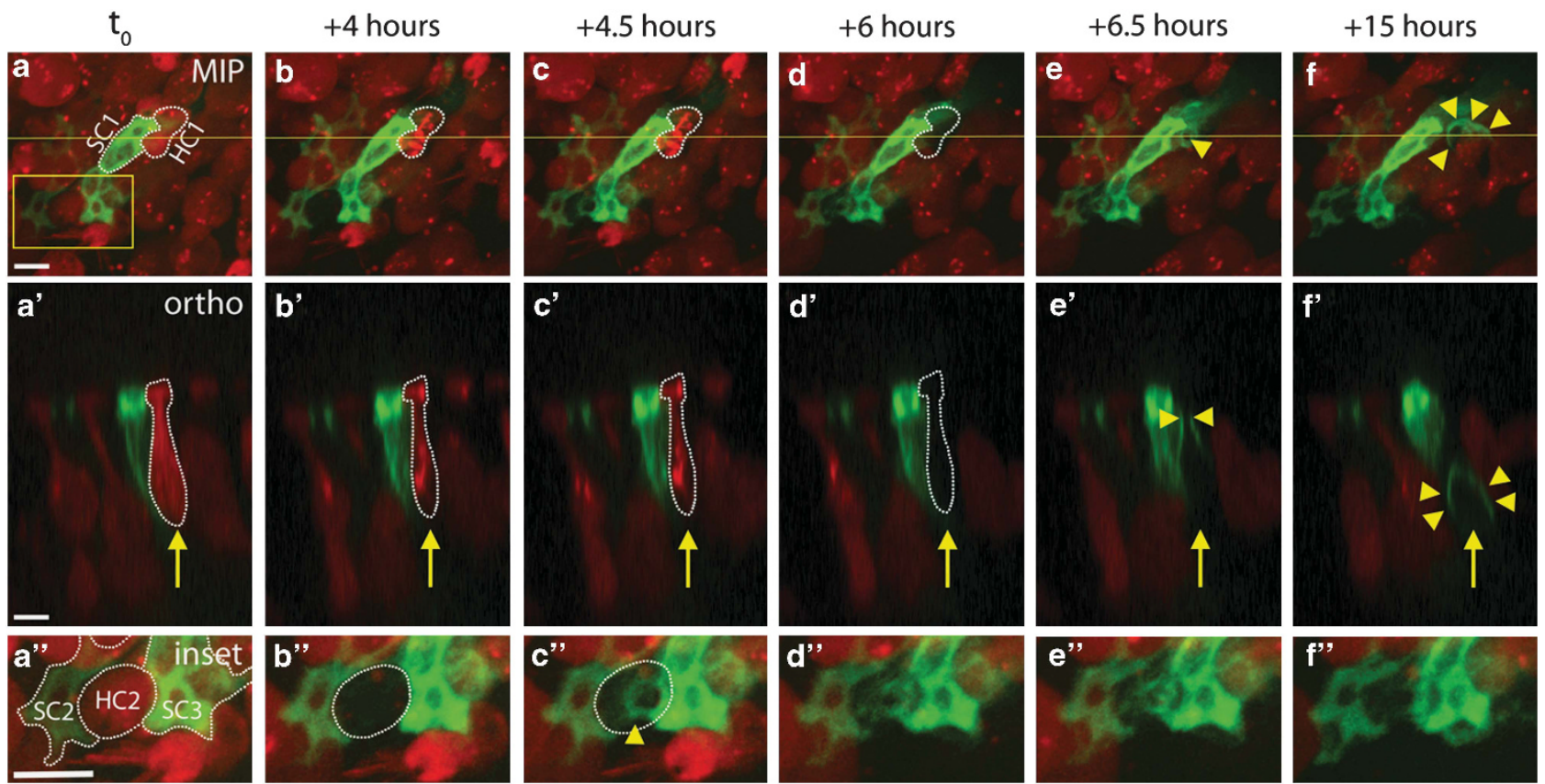

Figure 2 SCs constrict the apical portion of dead or dying HCs before removing them from the sensory epithelium. (a-f) In a series of MIPs taken from a 48-h live-imaging experiment, a single $\mathrm{HC}$ (red, labeled $\mathrm{HC1}$ in a) loses tdTomato signal at $+6 \mathrm{~h}$ (dashed outline in d). The neighboring green SC (labeled SC1 in a) is observed constricting the apical portion of $\mathrm{HC} 1$ within 30 min of tdTomato loss (single arrowhead in e). SC1 then forms a phagosome surrounding the remains of $\mathrm{HC} 1$, eliminating the $\mathrm{HC}$ corpse from the sensory epithelium (multiple arrowheads in f). $a^{\prime}-f^{\prime}$ Orthogonal views of the same imaging field as a-f, taken from the plane indicated by the yellow line in a. Arrow follows $\mathrm{HC1}$, as it loses tdTomato (dashed outline, d') before SC constriction (arrowheads, e') and phagosome formation (multiple arrowheads, f'). a"-f' Magnified inset views taken from the outlined box in a. Two SCs (labeled SC1 and SC2 in a") surround a single HC (labeled HC2 in a"), which loses tdTomato signal at +4 $\mathrm{h}$ (dashed outline, b"). SC2 and SC3 together constrict HC2 within $30 \mathrm{~min}\left(\mathbf{C}^{\prime \prime}\right)$. Movies are Supplementary Figures 2 and 3. Overall gain was increased on inset images. Scale bars $=5 \mu \mathrm{m}$ 
(Supplementary Figure 4), indicating that the dead $\mathrm{HC}$ occupies its original space until removal by the SC phagosome.

In live-imaging experiments in which HCs were killed by neomycin, initiation of the SC response immediately followed (or occurred at a time point indistinguishable from) loss of tdTomato from the HC. To examine whether these two events were separable in time, we decreased the imaging interval from 30 to $10 \mathrm{~min}$. Figure 3 a shows three views from the same image field (movies are Supplementary Figure 5). Figures 3a-i are $3 \mathrm{D}$ surface views of a red $\mathrm{HC}$ (dashed outline) and its neighboring green SC. Figures 3a'-i' are differential maximum intensity projections (MIPs), in which the change in signal relative to the previous frame is shown in white. Figures 3a"-i" show a 3D orthogonal view of the same area. In this series the $\mathrm{HC}$ loses tdTomato (dashed outline, Figures 3b, b' and b"), and within 10 min the SC has formed an F-actin-based process in direct apposition to the apical portion of the tdTomato-negative $\mathrm{HC}$ (arrowhead in Figure 3c"). Thirty minutes later, the SC processes have advanced toward the center of the dead $\mathrm{HC}$ and formed an apical constriction (arrowheads, Figures $3 f$ and f"). Although we cannot pinpoint the moment of bundle excision due to the loss of tdTomato in the $\mathrm{HC}$, the apical constriction appears to be tightly closed in Figures 3h-3h", with the phagosome structure visible $10 \mathrm{~min}$ later (arrowheads in Figure 3i"). Our data indicate that SCs first move to constrict neighboring $\mathrm{HCs}$ either in the same frame as $\mathrm{HC}$ death or later (Figure 3j). On average, SCs constricted the dead HC within $1 \mathrm{~h}$ of tdTomato loss (Figure 3k).

To examine the contents of the SC phagosome, TOTO-3 was used in longer-term live-imaging experiments. Figure 4 shows a series of $3 \mathrm{D}$ reconstructions (movie is Supplementary Figure 8 ) in which a $\mathrm{HC}$ dies (dashed outline, Figure 4c) and TOTO-3 enters the nucleus (arrowheads in Figure 4c, strongly

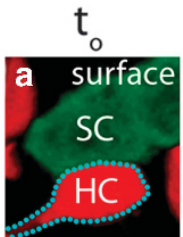

$+10 \mathrm{~min}$
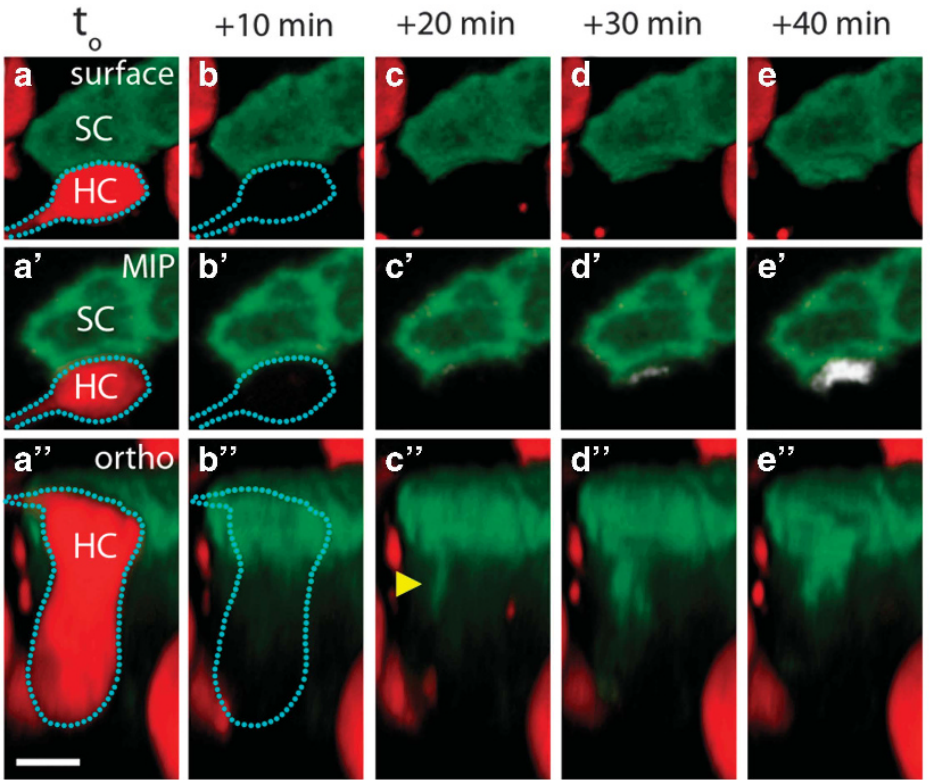

$+50 \mathrm{~min}$
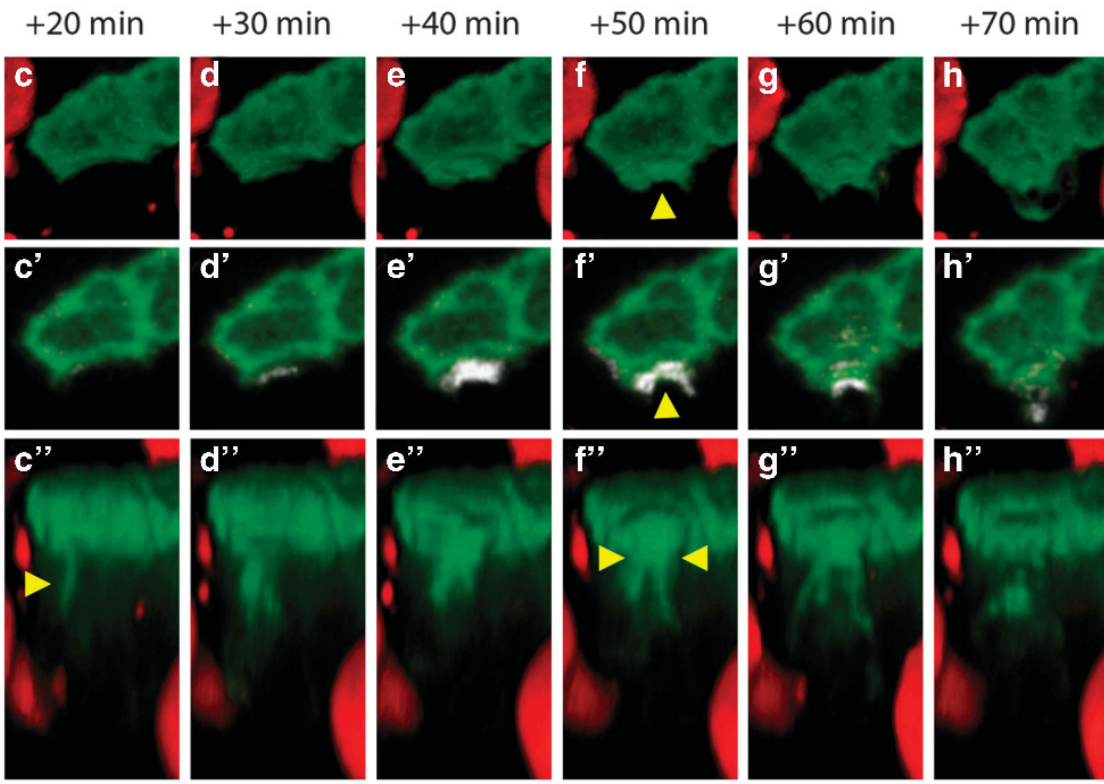

$+80 \min$

\section{j}

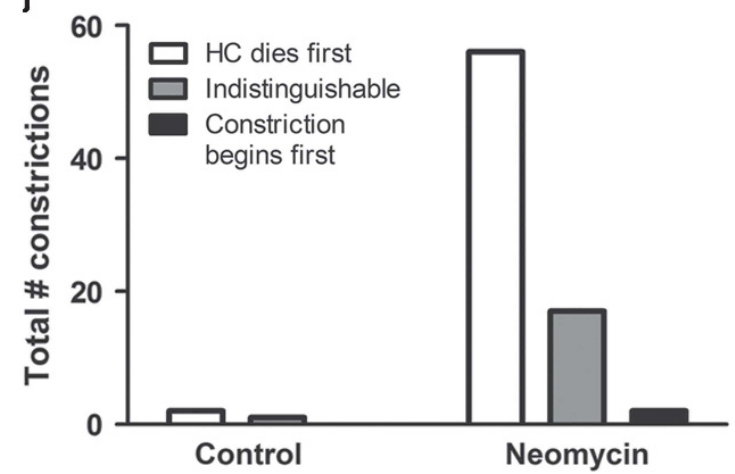

K

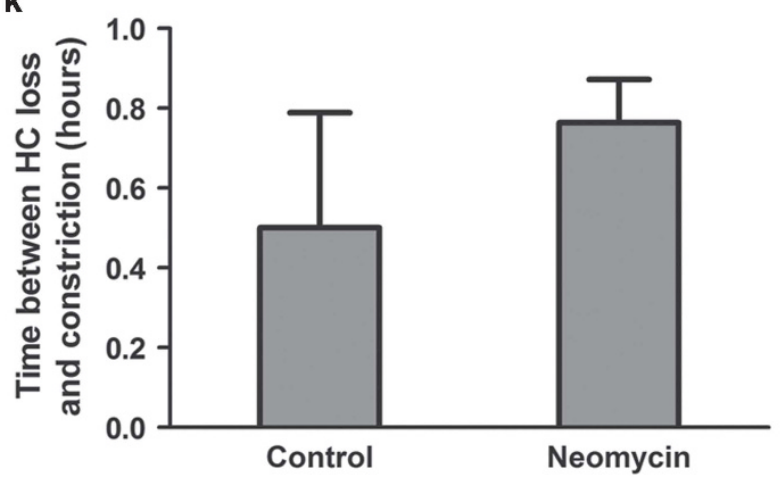

Figure $3 \mathrm{SC}$ constriction begins shortly after $\mathrm{HC}$ death. (a-i) 3D surface views of a single SC (SC, green) and neighboring HC (HC, red). (a'-i') MIP view of the same image field, containing a third channel (white) that displays the change in EGFP signal from the previous frame. a"--1" 3D orthogonal view of same HC and SC. The HC loses tdTomato signal (dashed outline in b-b") and the SC initiates constriction toward the HC within 10 min (arrowhead, $\left.\mathbf{c}^{\prime \prime}\right)$. Thirty minutes later, the apical constriction is visible (arrowheads, $\mathrm{f}-\mathrm{f}$ "), followed by the phagosome structure (arrowheads, i"). Scale bar $=5 \mu \mathrm{m}$. (j) Initiation of SC phagocytic activity occurs after or concurrent with HC death. Bars represent the sum total of all constrictions counted across four to five utricles per condition. (k) When SCs constricted dead or dying HCs, the time between the loss of HC membrane integrity and initiation of SC constriction of the apical portion of the $\mathrm{HC}$ averaged $<1 \mathrm{~h}$, regardless of the culture condition. Movies are Supplementary Figure 5 . $N=3-75$ SC constrictions across $4-5$ utricles per condition. Bars represent mean \pm S.E.M. 

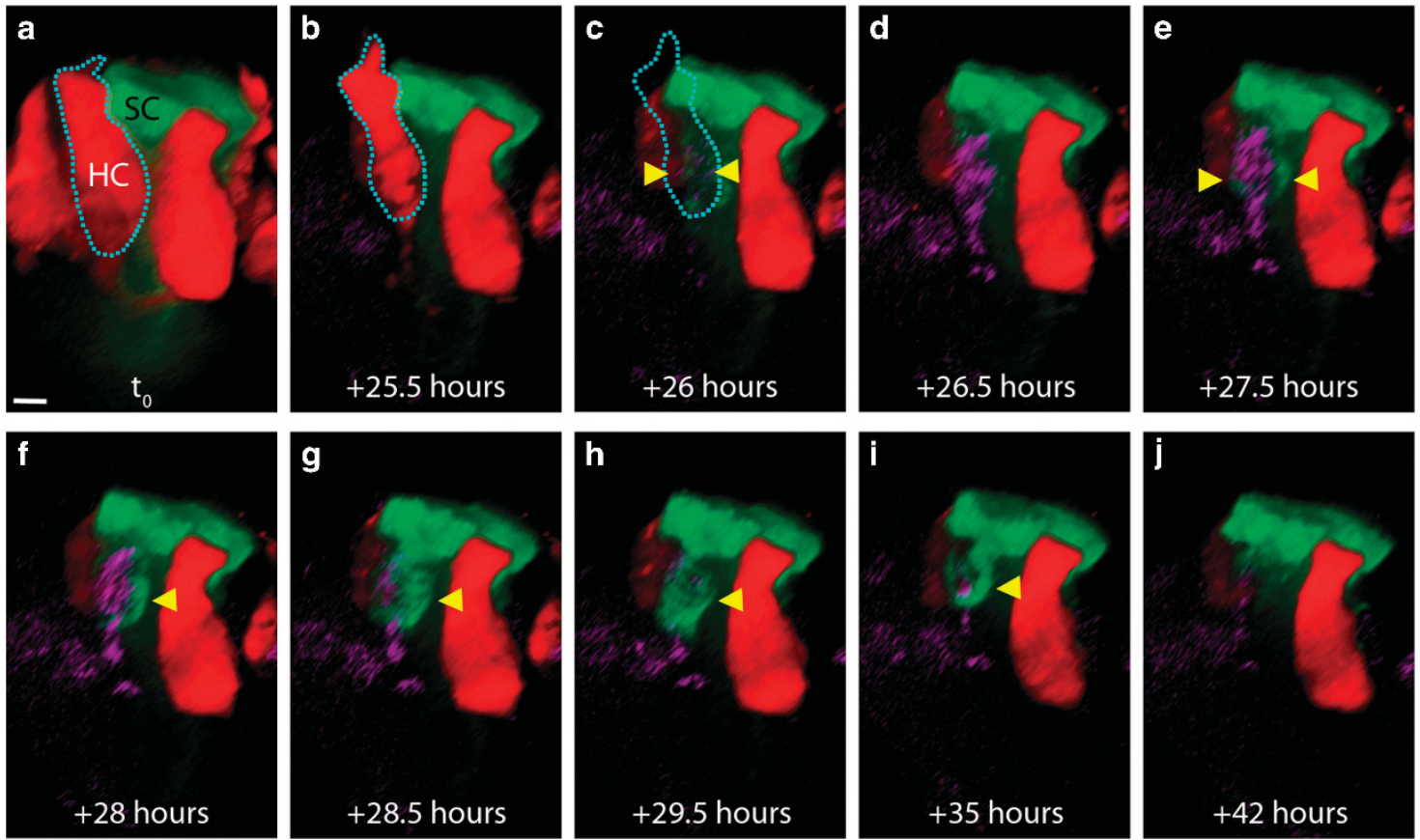

Figure 4 The SC phagosome contains the nucleus of a dead HC. (a and $\mathbf{b})$ 3D orthogonal view of a red HC and an adjacent green SC. (c) During live imaging, the HC loses tdTomato signal in the same frame in which TOTO-3 is weakly visible (arrowheads). (d) Thirty minutes later, the TOTO-3 is easily visible within the HC nucleus. (e): Within $1.5 \mathrm{~h}$ of tdTomato loss, the green SC has extended processes to surround the TOTO-3-positive nucleus (arrowheads). (f) A phagosome begins to surround the nucleus (arrowhead). $(\mathbf{g}$ and $\mathbf{h}$ ) The SC phagosome has fully surrounded the nucleus. ( $\mathbf{a}$ and $\mathbf{j})$ The HC has been degraded and the phagosome appears to have receded. Movie is Supplementary Figure 8 . Scale bar $=3.5 \mu \mathrm{m}$

visible in Figure 4d). Ninety minutes after $\mathrm{HC}$ death, the adjacent SC first extends processes toward the $\mathrm{HC}$ corpse (arrowheads, Figure 4e), which then further extend and fully surround the TOTO-3-positive nucleus as a phagosome (arrowhead, Figures $4 \mathrm{f}-\mathrm{h}$ ). The phagosome then gradually recedes (Figures $4 \mathrm{i}-\mathrm{j}$ ). These data confirm that the SC phagosome contains a dead $\mathrm{HC}$, and that SCs are actively removing $\mathrm{HC}$ corpses from the sensory epithelium.

SC phagocytic activity is reduced in cisplatin-treated utricles. We also examined SC phagocytic activity when HCs were killed by the other major ototoxic drug, cisplatin. The time courses of $\mathrm{HC}$ death are different for neomycinversus cisplatin-treated utricles: the average time required for $50 \%$ of the HCs to die was $23 \pm 6 \mathrm{~h}$ in neomycin and $45 \pm 3 \mathrm{~h}$ in cisplatin (mean \pm S.D.). To compare across conditions such that we were examining utricles with similar numbers of dead $\mathrm{HCs}$, we treated with each drug for $48 \mathrm{~h}$ and performed subsequent quantitative analyses relative to the point when $50 \pm 5 \%$ of the HCs had died (lost tdTomato signal). Neomycin-treated utricles exhibited a significant increase in the number of SC constrictions relative to controls. However, when utricles were treated with cisplatin, there was no increase in constrictions (Figure 5a). In addition, when 50\% of the HCs had died, those cultured in neomycin did not show any SC death, while those cultured in cisplatin exhibited nearly $50 \%$ SC death (Figure $5 b$ ).

After the stereocilia bundle is excised, SCs form actin-rich scars at the surface of the epithelium. ${ }^{2,12-17}$ We examined scar formation in fixed utricles after treatment with either neomycin or cisplatin. These utricles were not live-imaged but were cultured in neomycin or cisplatin until $50 \%$ of the $\mathrm{HCs}$ died, given an additional $4 \mathrm{~h}$ for the maximal SC response, and were fixed and stained for F-actin. In both control and neomycin-treated utricles, SC scars were consistently observed where HCs were missing (Figure 5c). In contrast, cisplatin-treated utricles revealed many dead $\mathrm{HCs}$ that were not covered by SC scars, a defect which persisted when utricles were cultured longer in cisplatin to allow for additional $\mathrm{HC}$ death and more time for SCs to respond (Supplementary Figure 9). Together, these data indicate that SCs are capable of robust scar formation in response to neomycin-induced $\mathrm{HC}$ death, while cisplatin-treated SCs show impaired scar-forming ability and an increase in cell death.

HC corpses accumulate in cisplatin-treated utricles.

As our experiments indicate that cisplatin impairs scar formation, we hypothesized that cisplatin treatment would also impair the clearance of HC corpses by SCs. We cultured utricles in neomycin or cisplatin until $50 \%$ of the $\mathrm{HCs}$ died and then allowed an additional $4 \mathrm{~h}$ for $\mathrm{HC}$ clearance. We then fixed the tissue and stained for myosin VIIA, a commonly used immunochemical marker that is specifically expressed in $\mathrm{HCs}^{28-32}$ We consistently observed tdTomato-negative (dead) HCs that showed immunoreactivity for myosin VIIA (Figure 6a). As our data indicate that loss of tdTomato is a reliable indicator of $\mathrm{HC}$ death, these tdTomato-negative/ myosin VIIA-positive HCs are corpses. Figure 6a shows control, neomycin-treated, and cisplatin-treated utricles stained for myosin VIIA. In merged images at the $\mathrm{HC}$ nuclear level (Figure 6a, merge), asterisks indicate all HC corpses. Merged images from the epithelial surface, the reticular 
a

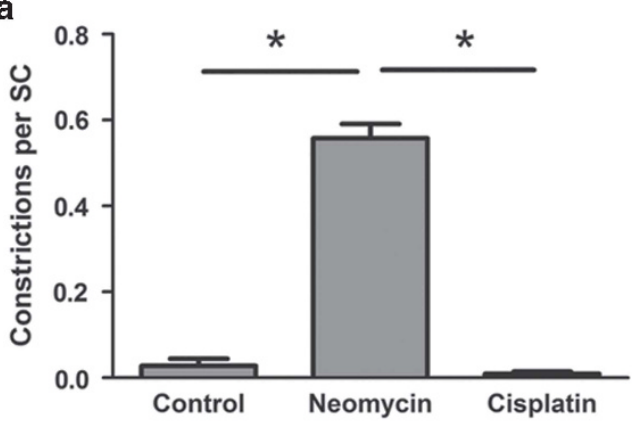

b

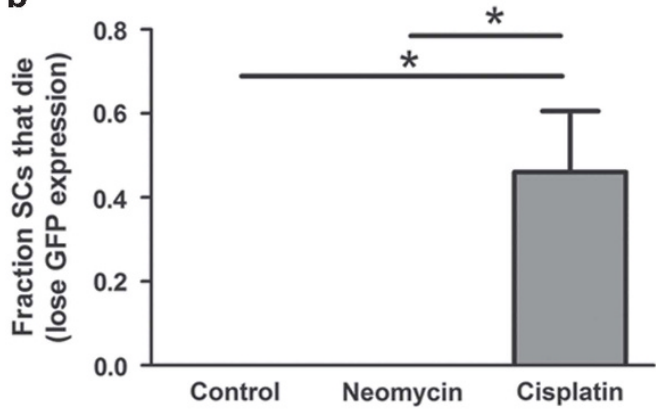

c
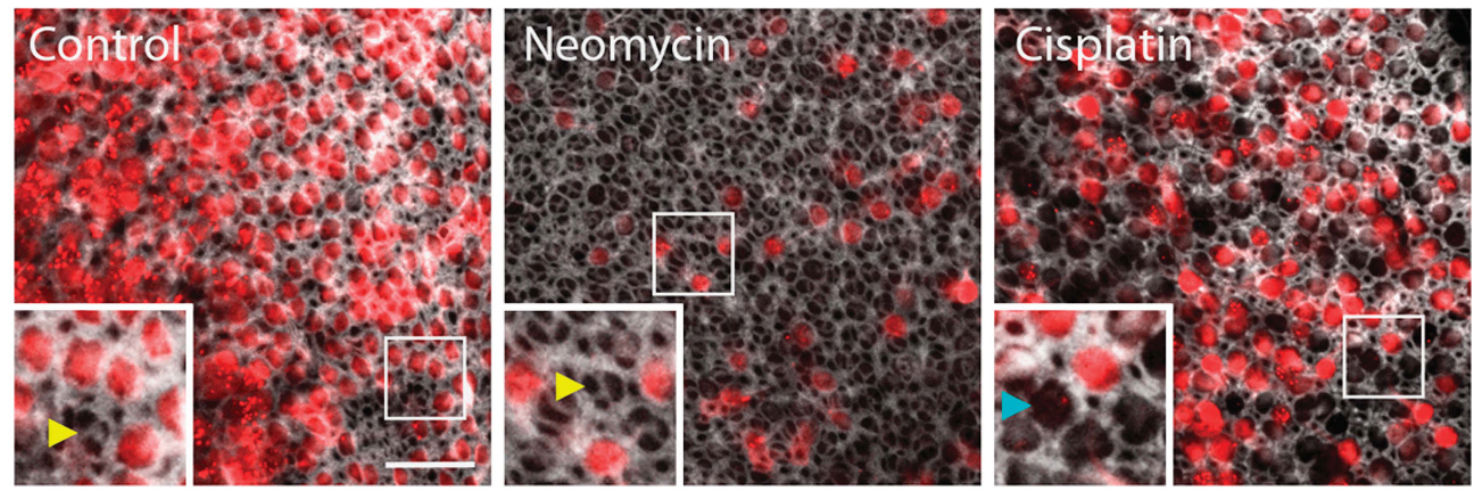

Figure 5 Cisplatin impairs SC phagocytic activity. Utricles were treated with either neomycin or cisplatin until $50 \%$ of the HCs died. (a) SCs in neomycin-treated utricles exhibit robust phagocytic activity, as indicated by the number of $\mathrm{SC}$ constrictions. This activity is significantly reduced when $\mathrm{HCs}$ are killed by cisplatin. $N=3-75 \mathrm{SC}$ constrictions across 4-5 utricles per condition. ${ }^{*} P<0.05$ versus neomycin. (b) When $50 \%$ of the HCs have died, no SCs have died in control or neomycin-treated utricles; however, cisplatin-treated utricles exhibit a significant increase in SC death (determined by complete loss of EGFP signal). Bars represent mean \pm S.E.M. $N=126-276$ SCs across 4-5 utricles per condition. ${ }^{*} P<0.05$ versus control. (c) In a separate set of experiments, utricles were not live-imaged, but were cultured until $50 \%$ of the HCs died, then fixed and stained with phalloidin (white). In a single plane taken beneath the stereocilia bundles, scars (yellow arrowheads, inset) are visible in both the control and neomycin-treated utricles in spaces where tdTomato-positive HCs (red) are missing. In cisplatin-treated utricles, spaces missing tdTomato-positive HCs were often not covered by SC scars (blue arrowhead, inset). Scale bar $=20 \mu \mathrm{m}$. Overall gain was increased on all inset images

lamina of the same image stacks (Figure 6a, final column) show the positions of the neighboring SCs (F-actin, white) relative to the myosin VIIA and tdTomato channels (individual channels, Supplementary Figure 10). HC corpses increased modestly in neomycin-treated utricles over controls. However, $\mathrm{HC}$ corpses in cisplatin-treated utricles increased significantly relative to neomycin-treated tissue (Figures $6 a$ and $b$ ). This difference in corpse accumulation is not attributable to a neomycin-induced reduction in myosin VIIA expression, as there was no difference in myosin VIIA signal intensity between living (tdTomato+) and dead (tdTomato-) HCs cultured in either neomycin or cisplatin (Figure 6c). Together, these data indicate that cisplatin impairs SC-mediated phagocytic removal of dead HCs.

HCs shrink before death. In addition to the differences we observed between cisplatin- and neomycin-treated tissue, we also observed similarities. We quantified $\mathrm{HC}$ volume in the hours before death (Figure 6d). In either neomycin or cisplatin, HCs exhibited similar decreases $\left(>150 \mu \mathrm{m}^{3}\right)$ in cell volume before losing tdTomato. In addition, there appears to be an increased rate of volume loss in the final 3-4 $\mathrm{h}$ before $\mathrm{HC}$ death. These data indicate that the HCs undergo a cell death program that begins hours before loss of membrane integrity, regardless of the ototoxic drug that induces $\mathrm{HC}$ death.

Cisplatin impairs the SC response to $\mathrm{HC}$ death in vivo. To investigate whether our ex vivo data reflect the mature mouse cochlea in vivo, we examined the $\mathrm{SC}$ responses to $\mathrm{HC}$ death in cochleas from adult mice that received systemic aminoglycosides or cisplatin. Figure 7 shows 3D renderings of F-actin (labeled with phalloidin) in cochleas from mice that were treated with saline, the aminoglycoside antibiotic kanamycin, or cisplatin. Cochleas from saline-treated mice showed normal cochlear architecture with three rows of outer $\mathrm{HCs}$ and a single row of inner HCs (Figure 7a). Cochleas from both kanamycin- and cisplatin-treated mice showed significant loss of outer HCs. Cochleas from kanamycintreated mice showed thick, F-actin-based scars at the reticular lamina (Figure $7 \mathrm{~b}$ ). However, the scars from cisplatin-treated mice were thinner and less robust (Figure 7c). Quantitative analysis of phalloidin signal intensity indicated that scars formed in response to cisplatin treatment contained significantly less F-actin than scars formed in response to aminoglycoside treatment (Figure 7d). These data indicate that the SC responses to $\mathrm{HC}$ death are also impaired by cisplatin in the mouse cochlea in vivo. 
a
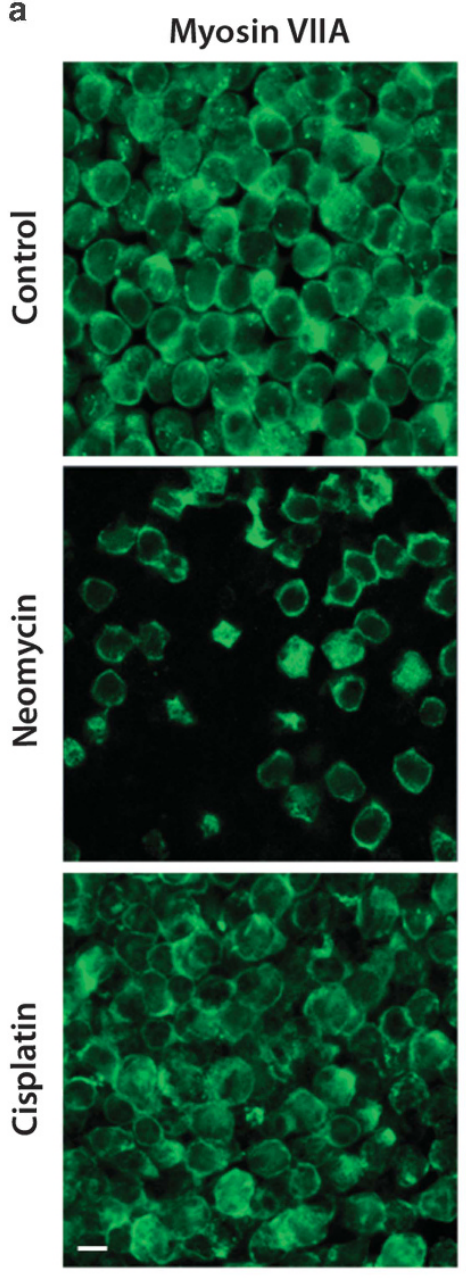

\section{b}

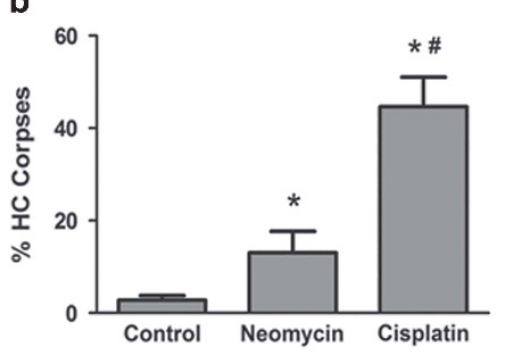

tdTomato
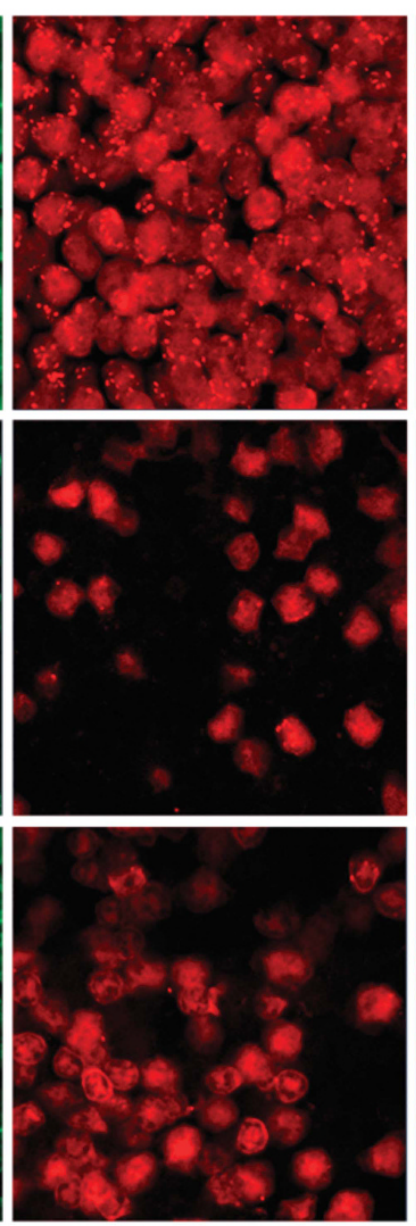

Merge
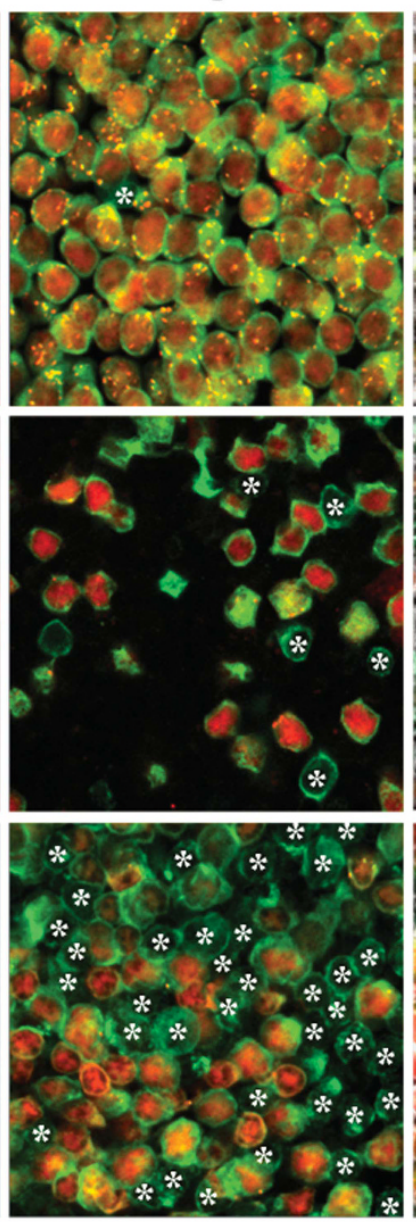

Reticular Lamina

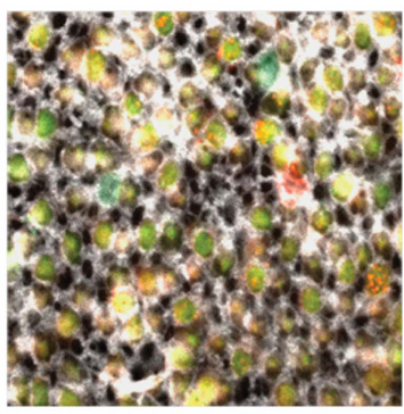

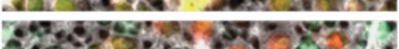

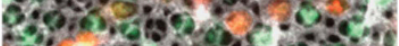

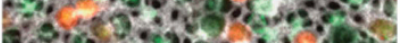
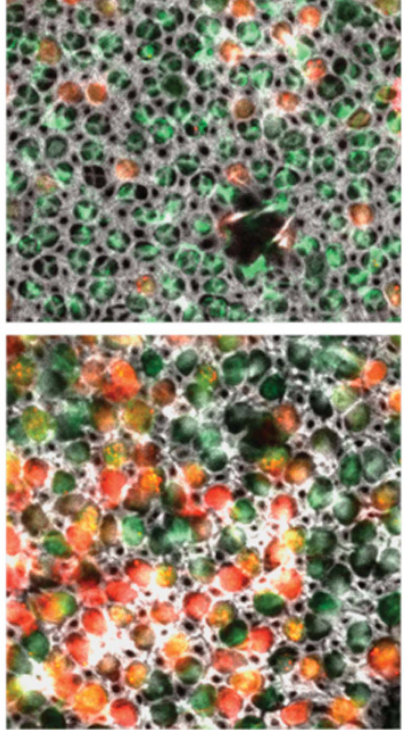

c

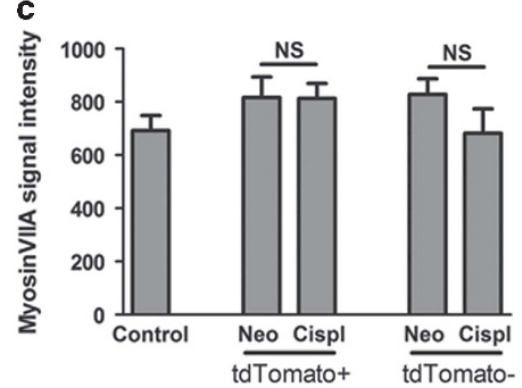

d

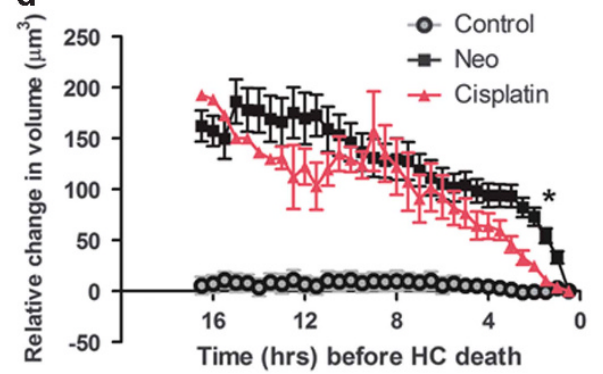

Figure $6 \mathrm{HC}$ corpses accumulate in cisplatin-treated utricles. (a) Utricles were cultured in either control medium (upper row), neomycin (middle row), or cisplatin (bottom row) until $50 \%$ of the $\mathrm{HCs}$ died (or a matched time point in control medium). Four hours later, utricles were fixed and stained for the HC marker myosin VIIA (green), which was compared with the tdTomato signal present in living HCs (red). HC corpses (cells that are myosin VIIA-positive and tdTomato-negative) are indicated by asterisks in merged images from each condition. All images were acquired at the plane of the $\mathrm{HC}$ nuclei, with the exception of those in the fourth column, which were taken at the level of the reticular lamina and include the f-actin signal (white) in the merged image. Scale bar $=5 \mu \mathrm{m}$. (b) The percentage of HC corpses was significantly increased in neomycin-treated utricles over controls, while the percentage of HC corpses was highest in the cisplatin-treated utricles. Bars represent mean \pm S.E.M. $N=358-2583 \mathrm{HCs}$ across $3-4$ utricles per condition. ${ }^{*} P<0.05$ versus control, ${ }^{\#} P<0.05$ versus neomycin. (c) Myosin VIIA signal intensity was not reduced in neomycin-treated tissue relative to cisplatin-treated tissue. Both tdTomato+ and tdTomato- HCs were analyzed at the nuclear plane. Bars represent mean \pm S.E.M. $N=7 \mathrm{HCs}$ per condition. (d) HC volume was analyzed over time in control, neomycin, and cisplatin-treated tissue. As the HCs cultured in either neomycin or cisplatin approached $\mathrm{HC}$ death (indicated by loss of tdTomato signal at $t=0 \mathrm{~h}$ ), $\mathrm{HCs}$ from both conditions shrank in volume, particularly in the last $3-4 \mathrm{~h}$ before death. $\mathrm{HCs}$ cultured in control medium were tracked for the same amount of time as the drug-treated utricles, but they did not die and did not shrink in volume. $N=1-31 \mathrm{HCs}$ per time point, values represent mean \pm S.E.M. ${ }^{*} P<0.0002$ via $t$-test with Bonferroni correction, neomycin versus cisplatin 

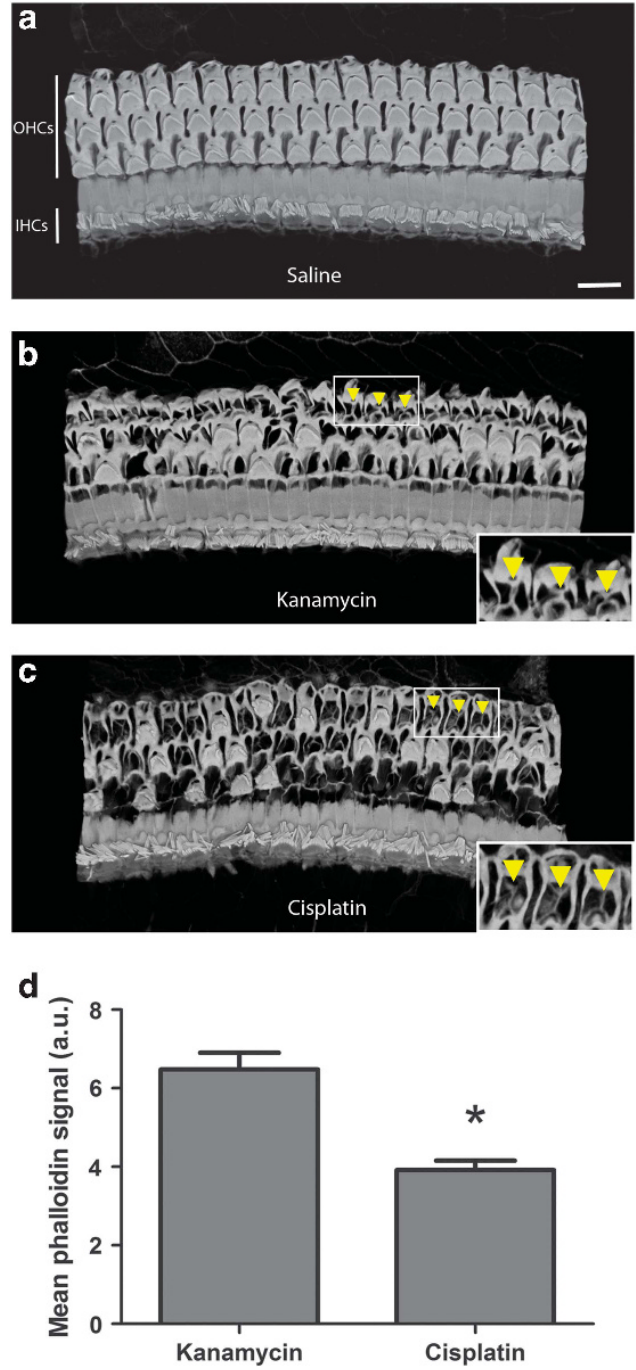

Figure 7 Cisplatin results in defects in SC phagocytic activity in mouse cochlea in vivo. (a) In cochleas taken from a saline-treated mouse and stained with phalloidin to label F-actin, a single row of inner $\mathrm{HCs}$ (IHCs) and three rows of outer $\mathrm{HCs}(\mathrm{OHCs}$ ) are present and have intact stereocilia bundles. Scale bar $=14 \mu \mathrm{m}$. (b) In cochleas of mice treated with systemic kanamycin, many dead OHCs have been replaced with actin-rich SC scars (a subset of which is highlighted by arrowheads). (c) In contrast, when mice were treated with cisplatin, the scars that replaced missing OHCs were thinner and composed of less actin (arrowheads) than those formed in cochleas of kanamycin-treated mice. Insets show magnified scars from $\mathrm{OHC}$ regions (arrowheads). (d) F-actin signal intensity was reduced in scars from cisplatin-treated mice relative to those of kanamycin-treated mice. $N=36-43$ scars across 3 cochleas per condition. ${ }^{*} P<0.05$ versus kanamycin-treated mice

\section{Discussion}

Our work examined the SC response to $\mathrm{HC}$ death caused by both major classes of ototoxic drugs. When HCs are killed by aminoglycoside antibiotics, our data are consistent with previous studies showing that SCs constrict the apical portion of HCs. ${ }^{13-15,17,33,34}$ Phagosome formation has previously been reported in immature avian utricles ${ }^{15}$ and our study is the first to use live imaging to examine the formation of basket-like, $\mathrm{F}$-actin-rich phagosomes in the mature mammalian inner ear. We followed individual HCs over time, as they lost membrane integrity, internalized TOTO-3, and were encapsulated and

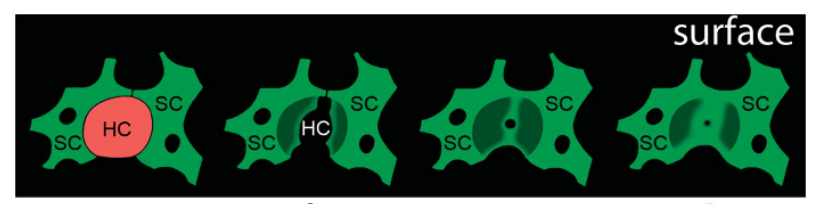

a

b

c

d

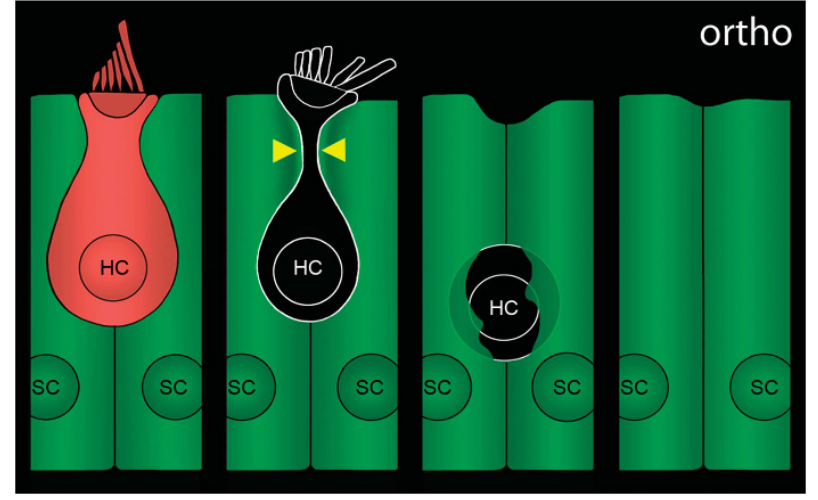

Figure 8 Model of SC phagocytic activity following HC death. (a) The architecture of a normal utricle includes SCs surrounding a healthy $\mathrm{HC}$ (surface view in top panel, orthogonal view in bottom panel). (b) Following treatment with aminoglycoside antibiotics, SCs constrict the $\mathrm{HC}$ beneath the cuticular plate (yellow arrowheads). Loss of tdTomato from the $\mathrm{HC}$ indicates loss of $\mathrm{HC}$ membrane integrity and death. (c) SC constriction results in excision of the stereocilia bundle and cuticular plate from the HC. SCs extend processes to form a phagosome around the remains of the dead HC. (d) In the final stage of the process, the HC corpse has been removed and the SCs have formed a scar at the reticular lamina

degraded by neighboring SCs. TOTO-3 labeled dead HCs following the loss of tdTomato, confirming that SC phagosomes contain nuclei of tdTomato-negative HC corpses. These data are supported by previous reports of $\mathrm{HC}$ nuclei or debris within SCs after damage. ${ }^{15,35-37}$

Our data are consistent with a model in which glia-like SCs remove dead $\mathrm{HCs}$ from the mature mammalian inner ear (Figure 8). SCs completely surround healthy HCs (Figure 8a). Following exposure to an ototoxic aminoglycoside antibiotic (i.e., neomycin), HCs lose membrane integrity (lose tdTomato) and SCs constrict dead HCs beneath the cuticular plate (Figure 8b, arrowheads). This constriction excises the stereocilia bundle and cuticular plate from the $\mathrm{HC}$, while SCs further constrict the apical portion of the dead $\mathrm{HC}$ and extend F-actin processes to form a phagosome around the HC corpse (Figure 8c). Our data suggest that the SCs ultimately remove the remains of the dead $\mathrm{HC}$ and form a scar at the reticular lamina (Figure 8d). Our experiments using TOTO-3 indicate that the dead $\mathrm{HC}$ nucleus remains fairly static, enclosed within an SC phagosome for several hours. This is a slower time course of engulfment and removal than was described in the chick $^{15}$ and it is consistent with observations regarding the enhanced responsiveness of chick versus mammalian SCs during wound healing. ${ }^{38}$ We also observed instances in which SCs ultimately push the $\mathrm{HC}$ corpse deeper into the epithelium, consistent with the idea that macrophages may also participate in the late stages of removal of dead HCs. ${ }^{39}$

SCs generally advanced toward the apex of the $\mathrm{HC}$ concurrently with or following $\mathrm{HC}$ death. This is in contrast with what has been reported in the developing chick, in which SCs constricted the apical portion of the $\mathrm{HC}$ before loss of 
membrane integrity and TOTO-3 uptake occurred concurrently with phagosome formation. ${ }^{15}$ The SC constriction in the embryonic chick was also faster than was observed here in the mammal. In the chick utricle, the constrictive event lasted $\sim 180 \mathrm{~s}$, as opposed to the 30 -min (or longer) constrictions we often observed in the adult mouse utricle. However, as $\sim 1 / 3$ of the constrictions in our study began in the same imaging frame in which the $\mathrm{HC}$ lost tdTomato, it was not possible to conclusively determine which event occurred first in those instances. This close timing between the loss of tdTomato and the constriction by the SC suggests that SCs were likely responding to $\mathrm{HC}$ death, possibly signaled by the release of intracellular ATP. ${ }^{4,40,41}$ It is also possible that HCs prime their surrounding SCs with a stress signal before they lose membrane integrity. The idea that HCs may signal their impending death is supported by our observation that dying HCs shrink for several hours before losing membrane integrity, a phenomenon that occurred in both neomycin- and cisplatintreated utricles. These data suggest that $\mathrm{HC}$ shrinkage occurred independently of SC activity, consistent with recent data reported in the mammalian cochlea. ${ }^{35}$ The slow shrinkage that precedes $\mathrm{HC}$ death may thus represent the initiation of a cell death program that began hours before any SC activity was observed.

Our data indicate that the SC phagocytic response, which is robust in aminoglycoside-treated tissue, is nearly absent in cisplatin-treated tissue. This suggests that either (1) the stress signal emitted from dying $\mathrm{HCs}$ is different in response to aminoglycosides versus cisplatin treatment or (2) the SCs themselves (or at least their phagocytic responses) were impaired by cisplatin. The number of apical constrictions was reduced to nearly zero in cisplatin-treated tissue. This finding, coupled with an increase in SC death, implies that SCs are impaired by cisplatin but not by aminoglycosides. Although the cisplatin-induced death of $\sim 50 \%$ of SCs would be expected to reduce $\mathrm{SC}$ activity, this loss does not account for the near-total loss of constrictions we observed in cisplatin-treated tissue. The surviving cisplatin-treated SCs were almost never observed constricting the apical portion of a $\mathrm{HC}$, despite being surrounded by the same amount of $\mathrm{HC}$ death as the neomycin-treated SCs. The idea that cisplatin specifically impairs SC activity is reinforced by previous work showing that cisplatin increased apoptosis in dissociated chick SC cultures, and it impaired regeneration and $\mathrm{HC}$ debris removal in the chick utricle. ${ }^{24}$ In addition, a recent study indicates that cisplatin (but not neomycin) damages cell-cell junctions ${ }^{42}$ and another study reported deficient scar formation in the adult mouse utricle upon treatment with Latrunculin A, which prevents actin polymerization. ${ }^{17}$ The resulting pattern at the reticular lamina reported in Burns and Corwin ${ }^{17}$ is reminiscent of that reported here following cisplatin treatment (Figure $5 \mathrm{c}$ ), consistent with cisplatin disrupting actin dynamics. ${ }^{43-45}$ Our in vivo data show that phalangeal scars formed in aminoglycoside-treated cochleas are more actin-rich than those formed in cisplatin-treated cochleas (Figure 8), further suggesting that cisplatin impairs F-actin dynamics in the inner ear.

We observed an accumulation of $\mathrm{HC}$ corpses within cisplatin-treated sensory epithelia. This corpse accumulation is consistent with both the lower level of SC phagocytic activity we observed and the increase in SC death. Accumulation of $\mathrm{HC}$ corpses has further implications for tissue health, as uncleared apoptotic cells would likely release proinflammatory intracellular contents into the surrounding tissue, causing secondary necrosis. ${ }^{46}$ In addition, our observation of tdTomato-negative/myosin VIIA-positive HC corpses highlights a caveat of using immunochemical $\mathrm{HC}$ markers, as a percentage of the labeled cells are likely to be corpses.

Data from our labs and others indicate that SCs have vital roles in determining whether HCs under stress ultimately live or die. The current study indicates that these cells are critical after HCs die. Reminiscent of the role microglia have in removing neuronal debris, ${ }^{47}$ SCs remove $\mathrm{HC}$ corpses and preserve the remaining health of the tissue. However, important questions remain about intercellular signals between dying $\mathrm{HCs}$ and their surrounding glia-like SCs, the role of resident macrophages, and exactly how long in advance of its death the HC begins to send 'distress signals'. Understanding intercellular signaling between these cell types will enhance our understanding of the roles of SCs and their responses to $\mathrm{HC}$ stress and death.

\section{Materials and Methods}

All reagents were obtained from Sigma Aldrich (St Louis, MO, USA), unless otherwise specified.

Utricle cultures. All mice were obtained from the Jackson Laboratory (Bar Harbor, ME, USA). The offspring resulting from a cross between B6.Cg- Tg

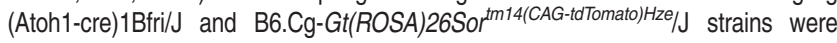
used for all in vitro experiments. The mice were euthanized by $\mathrm{CO}_{2}$ asphyxiation followed by decapitation; all animal procedures were approved by the NINDS/ NIDCD Animal Care and Use Committee. The adult mouse utricle preparation has been previously described in detail ${ }^{26,48}$ Briefly, temporal bones were removed from adult Atoh1-Cre x Rosa26-tdTomato transgenic mice of both sexes (4-12 weeks old) and placed in sterile M199 (Life Technologies Corporation, Carlsbad, CA, USA). Utricles were then dissected under sterile conditions and otoconia were removed with an eyelash tool (Ted Pella, Redding, CA, USA).

For experiments that did not require live imaging, utricles were then transferred to complete culture medium (DMEM/F12 (Life Technologies Corporation)) with $50 \mathrm{U} / \mathrm{ml}$ Penicillin $\mathrm{G}$ and $5 \%$ fetal bovine serum (Life Technologies Corporation) overnight at $37^{\circ} \mathrm{C}$ in a humidified atmosphere with $95 \%$ air $/ 5 \% \mathrm{CO}_{2}$. The following day $(\sim 20 \mathrm{~h}$ post dissection), utricles were treated with either $3 \mathrm{mM}$ neomycin sulfate or $30 \mu \mathrm{g} / \mathrm{ml}$ cisplatin (APP Pharmaceuticals, LLC, Schaumburg, IL, USA). Before addition to the culture medium, neomycin sulfate was equilibrated at $37^{\circ} \mathrm{C}, 95 \%$ air $/ 5 \% \mathrm{CO}_{2}$ for a minimum of $2 \mathrm{~h}$, and cisplatin was equilibrated for a minimum of $30 \mathrm{~min}$. Drugtreated utricles were cultured for the average amount of time necessary for $50 \%$ of the $\mathrm{HC}$ population to die, plus an additional $4 \mathrm{~h}$ to allow SCs time to clear dead HCs. This timing was calculated for each drug from a minimum of four separate liveimaging experiments. tdTomato-positive $\mathrm{HCs}$ were counted in the first frame of each experiment. When the frame contained $50 \pm 5 \%$ of the original number of tdTomatopositive $\mathrm{HCs}$, that time point was recorded and used to calculate the average time necessary for $50 \% \mathrm{HC}$ death, which was $23 \pm 6 \mathrm{~h}$ (mean \pm S.D.) in $3 \mathrm{mM}$ neomycin and $45 \pm 3 \mathrm{~h} \mathrm{in} \mu \mathrm{g} / \mathrm{ml}$ cisplatin.

Immunostaining and $\mathrm{HC}$ corpse quantification. At the end of the culture period, utricles were fixed for $30-40 \mathrm{~min}$ in $4 \%$ paraformaldehyde in $0.1 \mathrm{M}$ Sorensen's phosphate buffer (SPB) and washed in SPB. Tissue was blocked/ permeabilized for $3 \mathrm{~h}$ in $0.8 \%$ normal goat serum (Vector Laboratories, Burlingame, CA, USA), $0.4 \%$ Triton $X-100$, and $2 \%$ bovine serum albumin. Utricles were incubated overnight at $4{ }^{\circ} \mathrm{C}$ with a mouse monoclonal antibody against myosin VIIA (138-1, diluted 1:100 in blocking buffer, Developmental Studies Hybridoma Bank, lowa City, IA, USA). This antibody was detected using Alexa Fluor 488 goat antimouse IgG (Life Technologies Corporation). Following secondary antibody incubation, utricles were stained with Phalloidin-Atto 390 for $1 \mathrm{~h}$ before mounting onto glass slides with Fluoromount $\mathrm{G}$ (Southern Biotech, Birmingham, AL, USA). 
Images of the extrastriolar region within each utricle were acquired at $\times 40$ magnification on an LSM 780 confocal microscope (Carl Zeiss Microscopy, Oberkochen, Germany).

For quantification of $\mathrm{HC}$ corpses (HCs that were myosin VIIA-positive and tdTomato-negative), Zen 2012 software (Blue edition, Carl Zeiss Microscopy) was used. HCs were counted in $50 \times 50 \mu \mathrm{m}$ regions of interest (ROls), with a minimum of six ROls examined in the extrastriolar region of each utricle. HCs were counted in these ROls by an investigator who was blinded with respect to the tdTomato channel. Following these counts, the tdTomato channel was visually enabled and HCs that were negative for tdTomato (equivalent to background) were counted. Control utricles for each time point were quantified to determine average $\mathrm{HC}$ density, which was used as a reference point to ensure that each quantified area of the drug-treated utricles contained $50 \pm 25 \%$ of the control average $\mathrm{HC}$ density. This controlled for amount of $\mathrm{HC}$ death when quantifying the percentage of corpses in each area. Time-matched controls for each drug $(27 \mathrm{~h}$ in culture to match neomycin and $49 \mathrm{~h}$ in culture to match cisplatin, equivalent to the mean time to reach $50 \% \mathrm{HC}$ death in each drug-treated condition, plus $4 \mathrm{~h}$ for $\mathrm{HC}$ clearance) did not differ significantly from each other with respect to the percent of $\mathrm{HC}$ corpses, and so were statistically pooled into a single control condition.

In an additional set of experiments designed to maximize the chance of seeing $\mathrm{SC}$ respond to $\mathrm{HC}$ death caused by cisplatin, utricles were incubated for additional time in cisplatin relative to neomycin. In these experiments, utricles were cultured for $20 \mathrm{~h}$ in $3 \mathrm{mM}$ neomycin or $52 \mathrm{~h}$ in $30 \mu \mathrm{g} / \mathrm{ml}$ cisplatin, with time-matched controls. Following fixation, utricles were stained with anti-myosin VIIA and phalloidin. Myosin VIIA signal intensity was quantified in Volocity software (version 6.3, PerkinElmer, Waltham, MA, USA) by drawing a $12 \times 12 \times 10 \mu \mathrm{m}$ (66248 voxels) $\mathrm{ROI}$ at the $\mathrm{HC}$ nuclear level, detecting objects within 1 S.D. of the mean signal, and measuring fluorescent intensity of the objects within the ROI. Twenty-hour and 52-h controls were not significantly different from each other and so all control values were pooled.

Adenovirus infection and live imaging. Adenoviral infection of SCs in adult mouse utricles has been described. ${ }^{26,28}$ Following dissection and otoconia removal, utricles were transferred to a Nunc MicroWell MiniTray (Thermo Scientific, Waltham, MA, USA) and infected with a recombinant adenovirus (adenovirus type $5, \mathrm{DE} 1 / \mathrm{E} 3)$ carrying LifeAct-EGFP under the control of the human CMV promoter. Ad-LifeAct-EGFP was generated by sequencing and subcloning an $\sim 1.0-\mathrm{kb}$ Nhel/Notl fragment from the LifeAct-EGFP plasmid (kind gift from Guillaume Charras, UCL) into a Dual-CCM shuttle vector, which was then subcloned into an adenoviral backbone (Vector BioLabs, Philadelphia, PA, USA).

Ad-LifeAct-EGFP $\left(1.05 \times 10^{7}\right.$ plaque-forming units) was added to the utricles for $2 \mathrm{~h}$ in serum-free medium (DMEM with $50 \mathrm{U} / \mathrm{ml}$ Penicillin G). Following adenovirus infection, utricles were transferred to 24-well plates and maintained overnight in complete culture medium. For select experiments that were not used for quantification of apical constrictions, the virus-infected utricles were maintained in culture for up to 2 days before imaging.

Utricles were imaged on a spinning disk confocal microscope (Carl Zeiss Cell Observer SD) controlled by AxioVision software (Carl Zeiss Microscopy) or Zen 2012 (Black/Blue editions, Carl Zeiss Microscopy), using a Zeiss Plan-Apochromat, $\times 63$, 1.4 NA, oil objective that was heated to $37^{\circ} \mathrm{C}$ during acquisition. Laser lines $(488,561$, and $639 \mathrm{~nm})$ were used in conjunction with 525/50,617/17, and 690/50 nm emission filter sets, respectively. Images were acquired with either a Cool Snap HQ2 CCD camera (Photometrics, Tucson, AZ, USA) or an Evolve EMCCD camera (Photometrics). Imaging was performed at 10- or 30-min intervals for a maximum duration of $70 \mathrm{~h}$ in phenol red-free complete culture medium (control) or phenol redfree medium treated with either $3 \mathrm{mM}$ neomycin or $30 \mu \mathrm{g} / \mathrm{ml}$ cisplatin. All live-imaging data were obtained from the extrastriolar region. During imaging, cultures were maintained with $94 \%$ air $/ 6 \% \mathrm{CO}_{2}$ in a humidified atmosphere.

In each figure, image frames are denoted as occurring relative to $t_{0}$, which was selected relative to an event of interest and was a variable amount of time $(0-28 \mathrm{~h})$ after imaging began. Supplementary Movies and figure legends accompany each figure that included live imaging and provide details regarding the timing of each $t_{0}$. In calculating the differential MIP in Figure 3, the image stack was cropped to the upper $5 \mu \mathrm{m}$ of the reticular lamina before the differential channel was created.

Loss of $\mathrm{HC}$ membrane integrity. To assess the timing of the loss of $\mathrm{HC}$ membrane integrity relative to loss of tdTomato signal, utricles were infected with Ad-LifeAct-EGFP as above and imaged at 10-min intervals for $16 \mathrm{~h}$ in $3 \mathrm{mM}$ neomycin. After $19 \mathrm{~h}$ in culture, $1 \mu \mathrm{M}$ TOTO-3 iodide (Life Technologies Corporation) was added (delivered in $1 \mathrm{ml}$ phenol red-free complete culture medium, reducing the concentration of neomycin to $2 \mathrm{mM}$ ). Following TOTO-3 addition, imaging was resumed at $10 \mathrm{~min}$ intervals for $2 \mathrm{~h}$. Nuclear TOTO-3 signal was quantified using Volocity software by measuring fluorescent intensity within a $1.74 \times 1.74 \times 2.25 \mu \mathrm{m}$ (867 voxels) ROI within a selected nucleus. A blank region outside of the sensory epithelium was used to determine the background signal, which was subtracted from all intensity values.

To track TOTO-3-positive nuclei over longer periods, $0.5 \mu \mathrm{M}$ TOTO-3 iodide was added to the culture medium at the onset of the experiment and utricles were imaged at 30-min intervals.

HC volume. HC volume was quantified using Volocity software. Measurement settings were configured to automatically detect $\mathrm{HCs}$ as individual objects within the tdTomato channel; the accuracy of the automatic detection was visually verified and total cellular volume was calculated for each cell at each time point. Once determined for each cell, the automatic detection threshold settings were not changed over the duration of the time series.

Quantification of SC constrictions and death. Image stacks were visually analyzed in Zen 2012 and SC constrictions were counted. The criteria used to define an SC constriction were as follows: (1) the SC'S F-actin processes advanced toward or around the apical portion of the $\mathrm{HC}$; (2) when the process lasted for multiple frames, there was a clear progression in movement in which the SC's processes advanced toward the center of the $\mathrm{HC}$; and (3) the $\mathrm{HC}$ began with and subsequently lost tdTomato signal at some point during the imaging. The total number of constrictions over time (up to the point when $50 \%$ of the HCs had died) in each image set was normalized to the number of Ad-LifeAct-EGFP-infected SCs in the frame to account for any variability in SC infection efficiency. When examining the timing of the constrictions in MIPs of all movies, the beginning of the constriction was defined as the image frame in which the LifeAct-EGFP-positive F-actin processes were first observed to be extending toward or around the HC. SC death was indicated by the complete loss of EGFP signal.

In-vivo experiments. $\mathrm{CBA} / \mathrm{J}$ mice of both sexes were obtained from the Jackson Laboratory and used for all in-vivo experiments. Mice were 2 months old at the beginning of each injection protocol, which has been described. ${ }^{49}$ Briefly, cisplatin (APP Pharmaceuticals, LLC) was administered via intraperitoneal injection, $4 \mathrm{mg} / \mathrm{kg}$ each day for 4 days, followed by 10 days of recovery. Three such cycles of cisplatin were administered. Saline was administered in control mice. Mice were euthanized by $\mathrm{CO}_{2}$ asphyxiation, followed by decapitation, at the end of the third cycle.

Kanamycin sulfate was dissolved in sterile saline (Hospira, Inc., Lake Forest, IL, USA), to a final concentration of $45 \mathrm{mg} / \mathrm{ml}$. Kanamycin $(750 \mathrm{mg} / \mathrm{kg}$ ) was injected subcutaneously twice daily for 14 days. Mice recovered for 21 days after the final kanamycin injection. Saline was administered subcutaneously in control mice. Preand post-injection auditory brainstem response (ABR) testing was performed to examine hearing sensitivity in both cisplatin- and kanamycin-treated groups, as described. ${ }^{49}$ ABR testing was also performed for each control group.

Preparation of whole-mount cochleas. Cochleas were fixed overnight at $4{ }^{\circ} \mathrm{C}$ with ice-cold $4 \%$ paraformaldehyde after creating a small hole at the apex and the round window. The tissues were washed in PBS and decalcified in 0.5 M EDTA for 3-4 days. Cochleas were micro-dissected into three turns (apical, middle, and basal) and stained with Phalloidin-Atto 390. Each cochlear turn was mounted on a glass slide using Fluoromount G. F-actin staining was detected using the 405-nm laser line in conjunction with the $421-490 \mathrm{~nm}$ emission filter set. Images(x63) of selected regions from the transition zones (areas containing both intact and missing outer HCs) of the cochlea were taken using an LSM 780 confocal microscope (Carl Zeiss Microscopy). Imaging parameters were kept constant for all comparisons across groups.

Image stacks were visualized in Volocity software and the phalloidin signal was quantified by drawing a cylindrical $\mathrm{ROI}$ with a diameter of $3.95 \mu \mathrm{m}$ and a height of $5.1 \mu \mathrm{m}$ (11696 voxels). The ROls were each placed within regions of individual missing outer $\mathrm{HCs}$, centered on the reticular lamina, and fluorescent intensity within the ROI was measured.

Statistics. All statistics were performed in Prism 5 (Graphpad Software, Inc., La Jolla, CA, USA). In comparing two conditions, data were subjected to a Student's $t$-test. Experiments that required analysis of more than two conditions were 
subjected to ANOVA with Tukey's post-hoc test, with $P$-values $<0.05$ considered significant.

\section{Conflict of Interest}

The authors declare no conflict of interest.

Acknowledgements. We thank Dr Joseph Burns and Dr Jonathan Bird for their insightful comments on this manuscript. We also thank the NIDCD/NINDS animal care staff for their support during the cisplatin injection protocol, as well as Dr Jane Johnson at the University of Texas Southwestern Medical Center for generously providing the Atoh1-nGFP mice. This work was supported by the NIDCD Division of Intramural Research (ZIA DC000079) and a Flexigrant from Action on Hearing Loss.

1. World Health Organization. Deafness and hearing loss (web page), 2014. Available from http://www.who.int/mediacentre/factsheets/ss300/en/.

2. Raphael Y, Altschuler RA. Reorganization of cytoskeletal and junctional proteins during cochlear hair cell degeneration. Cell Motil Cytoskeleton 1991; 18: 215-227.

3. Hawkins Jr JE, Johnsson LG, Stebbins WC, Moody DB, Coombs SL. Hearing loss and cochlear pathology in monkeys after noise exposure. Acta Otolaryngol 1976; 81: 337-343.

4. Monzack EL, Cunningham LL. Lead roles for supporting actors: critical functions of inner ear supporting cells. Hear Res 2013; 303: 20-29.

5. Stankovic K, Rio C, Xia A, Sugawara M, Adams JC, Liberman MC et al. Survival of adult spiral ganglion neurons requires erbB receptor signaling in the inner ear. J Neurosci 2004; 24: 8651-8661.

6. Zuccotti A, Kuhn S, Johnson SL, Franz C, Singer W, Hecker D et al. Lack of brain-derived neurotrophic factor hampers inner hair cell synapse physiology, but protects against noiseinduced hearing loss. J Neurosci 2012; 32: 8545-8553.

7. Glowatzki E, Cheng N, Hiel H, Yi E, Tanaka K, Ellis-Davies GC et al. The glutamateaspartate transporter GLAST mediates glutamate uptake at inner hair cell afferent synapses in the mammalian cochlea. J Neurosci 2006; 26: 7659-7664.

8. Cotanche DA. Regeneration of hair cell stereociliary bundles in the chick cochlea following severe acoustic trauma. Hear Res 1987; 30: 181-195.

9. Corwin JT, Cotanche DA. Regeneration of sensory hair cells after acoustic trauma. Science 1988; 240: 1772-1774.

10. Cruz RM, Lambert PR, Rubel EW. Light microscopic evidence of hair cell regeneration after gentamicin toxicity in chick cochlea. Arch Otolaryngol Head Neck Surg 1987; 113: 1058-1062.

11. Ryals BM, Rubel EW. Hair cell regeneration after acoustic trauma in adult Coturnix quail. Science 1988; 240: 1774-1776.

12. Forge A. Outer hair cell loss and supporting cell expansion following chronic gentamicin treatment. Hear Res 1985; 19: 171-182.

13. Raphael Y, Altschuler RA. Scar formation after drug-induced cochlear insult. Hear Res 1991 51: 173-183.

14. Hordichok AJ, Steyger PS. Closure of supporting cell scar formations requires dynamic actin mechanisms. Hear Res 2007; 232: 1-19.

15. Bird JE, Daudet N, Warchol ME, Gale JE. Supporting cells eliminate dying sensory hair cells to maintain epithelial integrity in the avian inner ear. J Neurosci 2010; 30: 12545-12556.

16. Anttonen T, Kirjavainen A, Belevich I, Laos M, Richardson WD, Jokitalo E et al. Cdc42 dependent structural development of auditory supporting cells is required for wound healing at adulthood. Sci Rep 2012; 2: 978

17. Burns JC, Corwin JT. Responses to cell loss become restricted as the supporting cells in mammalian vestibular organs grow thick junctional actin bands that develop high stability. $J$ Neurosci 2014; 34: 1998-2011.

18. Wangemann $P$. Supporting sensory transduction: cochlear fluid homeostasis and the endocochlear potential. J Physiol 2006; 576: 11-21.

19. Li-Korotky HS. Age-related hearing loss: quality of care for quality of life. Gerontologist 2012; 52: 265-271.

20. Seixas NS, Neitzel R, Stover B, Sheppard L, Feeney P, Mills D et al. 10-Year prospective study of noise exposure and hearing damage among construction workers. Occup Environ Med 2012; 69: 643-650.

21. Cheng AG, Johnston PR, Luz J, Uluer A, Fligor B, Licameli GR et al. Sensorineural hearing loss in patients with cystic fibrosis. Otolaryngol Head Neck Surg 2009; 141: 86-90.
22. Hinojosa R, Riggs LC, Strauss M, Matz GJ. Temporal bone histopathology of cisplatin ototoxicity. Am J Otol 1995; 16: 731-740.

23. McDowell B, Davies S, Forge A. The effect of gentamicin-induced hair cell loss on the tight junctions of the reticular lamina. Hear Res 1989; 40: 221-232.

24. Slattery EL, Warchol ME. Cisplatin ototoxicity blocks sensory regeneration in the avian inner ear. J Neurosci 2010; 30: 3473-3481.

25. Becker B, Clapper J, Harkins KR, Olson JA. In situ screening assay for cell viability using a dimeric cyanine nucleic acid stain. Anal Biochem 1994; 221: 78-84.

26. Brandon CS, Voelkel-Johnson C, May LA, Cunningham LL. Dissection of adult mouse utricle and adenovirus-mediated supporting-cell infection. J Vis Exp 2012; 61: e3734.

27. Riedl J, Crevenna AH, Kessenbrock K, Yu JH, Neukirchen D, Bista M et al. Lifeact: a versatile marker to visualize F-actin. Nat Methods 2008; 5: 605-607.

28. May LA, Kramarenko II, Brandon CS, Voelkel-Johnson C, Roy S, Truong $\mathrm{K}$ et al. Inner ear supporting cells protect hair cells by secreting HSP70. J Clin Invest 2013; 123: $3577-3587$.

29. Hasson T, Heintzelman MB, Santos-Sacchi J, Corey DP, Mooseker MS. Expression in cochlea and retina of myosin VIla, the gene product defective in Usher syndrome type 1B. Proc Natl Acad Sci USA 1995; 92: 9815-9819.

30. Sahly I, El-Amraoui A, Abitbol M, Petit C, Dufier JL. Expression of myosin VIIA during mouse embryogenesis. Anat Embryol 1997; 196: 159-170.

31. Hasson T, Gillespie PG, Garcia JA, MacDonald RB, Zhao Y, Yee AG et al. Unconventional myosins in inner-ear sensory epithelia. J Cell Biol 1997; 137: 1287-1307.

32. Sergeyenko Y, Lall K, Liberman MC, Kujawa SG. Age-related cochlear synaptopathy: an early-onset contributor to auditory functional decline. J Neurosci 2013; 33: 13686-13694.

33. Gale JE, Meyers JR, Periasamy A, Corwin JT. Survival of bundleless hair cells and subsequent bundle replacement in the bullfrog's saccule. J Neurobiol 2002; 50: 81-92.

34. Baird RA, Burton MD, Lysakowski A, Fashena DS, Naeger RA. Hair cell recovery in mitotically blocked cultures of the bullfrog saccule. Proc Natl Acad Sci USA 2000; 97 11722-11729.

35. Anttonen T, Belevich I, Kirjavainen A, Laos M, Brakebusch C, Jokitalo E et al. How to bury the dead: elimination of apoptotic hair cells from the hearing organ of the mouse. $J$ Assoc Res Otolaryngol 2014; 15: 975-992.

36. Li L, Nevill G, Forge A. Two modes of hair cell loss from the vestibular sensory epithelia of the guinea pig inner ear. J Comp Neurol 1995; 355: 405-417.

37. Abrashkin KA, Izumikawa M, Miyazawa T, Wang CH, Crumling MA, Swiderski DL et al. The fate of outer hair cells after acoustic or ototoxic insults. Hear Res 2006; 218: 20-29.

38. Collado MS, Burns JC, Meyers JR, Corwin JT. Variations in shape-sensitive restriction points mirror differences in the regeneration capacities of avian and mammalian ears. PLoS One 2011; 6: e23861.

39. Kaur T, Hirose K, Rubel EW, Warchol M. Macrophage recruitment and epithelial repair following hair cell injury in the mouse utricle. Front Cell Neurosci 2015; doi:10.3389/fncel.2015.00150.

40. Gale JE, Piazza V, Ciubotaru CD, Mammano F. A mechanism for sensing noise damage in the inner ear. Curr Biol 2004; 14: 526-529.

41. Anselmi F, Hernandez VH, Crispino G, Seydel A, Ortolano S, Roper SD et al. ATP release through connexin hemichannels and gap junction transfer of second messengers propagate Ca2+ signals across the inner ear. Proc Natl Acad Sci USA 2008; 105: 18770-18775.

42. Slattery EL, Oshima K, Heller S, Warchol ME. Cisplatin exposure damages resident stem cells of the mammalian inner Ear. Dev Dyn 2014; 243: 1328-1337.

43. Kruidering M, van de Water B, Zhan Y, Baelde JJ, Heer E, Mulder GJ et al. Cisplatin effects on $\mathrm{F}$-actin and matrix proteins precede renal tubular cell detachment and apoptosis in vitro. Cell Death Differ 1998; 5: 601-614.

44. Arany I, Clark JS, Reed DK, Ember I, Juncos LA. Cisplatin enhances interaction between p66Shc and HSP27: its role in reorganization of the actin cytoskeleton in renal proximal tubule cells. Anticancer Res 2012; 32: 4759-4763.

45. Zeng $\mathrm{HH}$, Wang $\mathrm{K}$, Wang $\mathrm{B}$, Zhang $\mathrm{Y}$. Studies on the thermokinetic characterisation of actin polymerization and the effect of cisplatin. In J Biol Macromol 1996; 18: 161-166.

46. Silva MT. Secondary necrosis: the natural outcome of the complete apoptotic program. FEBS Lett 2010; 584: 4491-4499.

47. Petersen MA, Dailey ME. Diverse microglial motility behaviors during clearance of dead cells in hippocampal slices. Glia 2004; 46: 195-206.

48. Cunningham LL.. The adult mouse utricle as an in vitro preparation for studies of ototoxicdrug-induced sensory hair cell death. Brain Res 2006; 1091: 277-281.

49. Roy S, Ryals MM, Van den Bruele AB, Fitzgerald TS, Cunningham LL. Sound preconditioning therapy inhibits ototoxic hearing loss in mice. J Clin Invest 2013; 123: 4945-4949.

\section{Supplementary Information accompanies this paper on Cell Death and Differentiation website (http://www.nature.com/cdd)}

